Research Paper

\title{
Polymorphisms in the PVT1 Gene and Susceptibility to the Lung Cancer in a Chinese Northeast Population: a Case-control Study
}

\author{
Ziwei Zhang1,2, Hang Li1,2, Juan Li1,2, Xiaoting Lvi,2, Zitai Yang1,2, Min Gao ${ }^{1,2}$, Yanhong Bi, ${ }^{1,2}$, Shengli \\ Wang ${ }^{1,2}$, Zhigang Cui ${ }^{3}$, Baosen Zhou ${ }^{1,2}$, Zhihua Yin ${ }^{1,2}$ \\ 1. Department of Epidemiology, School of Public Health, China Medical University, Shenyang 110122, PR China; \\ 2. Key Laboratory of Cancer Etiology and Intervention, University of Liaoning Province, Shenyang 110122, PR China; \\ 3. School of Nursing, China Medical University, Shenyang 110122, China. \\ $\square$ Corresponding author: Zhihua Yin, Department of Epidemiology, School of Public Health, China Medical University; Key Laboratory of Cancer Etiology and \\ Intervention, University of Liaoning Province. No.77 Puhe Road, Shenyang North New Area, Shenyang 110122, People's Republic of China. E-mail: \\ zhyin@cmu.edu.cn. Telephone number: 18900910228. \\ (1) The author(s). This is an open access article distributed under the terms of the Creative Commons Attribution License (https://creativecommons.org/licenses/by/4.0/). \\ See http://ivyspring.com/terms for full terms and conditions.
}

Received: 2019.02.21; Accepted: 2019.09.30; Published: 2020.01.01

\begin{abstract}
Background: Long non-coding RNA (IncRNA) PVTI has been identified to be related to risk of a variety of cancers, such as gastric cancer, pancreatic cancer and follicular lymphoma. This study assesses the association between genetic polymorphisms of PVTI and the susceptibility to lung cancer as well as gene-environmental interaction.

Method: A hospital-based case-control study, including 515 lung cancer patients and 582 healthy controls, was carried out in Shenyang, China. Unconditional logistic regression analyses calculated the odds ratios (ORs) and their $95 \%$ confidence intervals $(\mathrm{Cls})$ to assess the associations between polymorphisms of rs2608053, rs1561927, rs13254990 and susceptibility to lung cancer. The gene-environment interaction was evaluated by additive model and multiplicative model.

Results: There were no statistically significant associations between rs2608053 and rs1561927 polymorphisms in PVT1 and risk of lung cancer in the overall population. The relationship between polymorphism rs 13254990 in PVT1 gene and lung adenocarcinoma was significant. Composed with individuals carrying CC genotypes, TT genotype carriers were more likely to develop lung adenocarcinoma (adjusted $\mathrm{OR}=2.095 ; 95 \% \mathrm{Cl}=1.084-4.047, \mathrm{P}=0.028$ ). In the recessive model, it also showed a statistically significant difference (TT vs CT+CC: adjusted $O R=2.251,95 \% \mathrm{Cl}=1.174-4.318$, $\mathrm{P}=0.015$ ). In nonsmokers, individuals carrying genotype $\mathrm{CT}$ had a lower risk of lung cancer than those with $C C$ genotype (adjusted $\mathrm{OR}=0.673,95 \% \mathrm{Cl}=0.472-0.959, \mathrm{P}=0.028$ ). Comparing with the homozygous CC, the patients with the heterozygous CT had a lower risk of NCSLC in the non-smoking group (adjusted $\mathrm{OR}=0.685,95 \% \mathrm{Cl}=0.477-0.984, \quad \mathrm{P}=0.040$ ). Additionally, gene-environment interaction results were not statistically significant in either additive model or multiplicative model.
\end{abstract}

Conclusion: The polymorphism rs13254990 in PVT1 gene is associated with the risk of lung adenocarcinoma in a Chinese northeast population.

Key words: lung cancer, lncRNA, PVT1, single nucleotide polymorphism, susceptibility

\section{Introduction}

As a heterogeneous, complex and intractable malignant tumor, lung cancer has brought enormous families' economic burden and jeopardized social health, which has gradually become one of the most important public health problems worldwide. According to the GLOBOCAN 2018 estimates of 
cancer incidence and mortality by the International Agency for Research on Cancer, lung cancer is the leading cause of cancer deaths both in China and the world[1]. Data indicated that an estimated 2.1 million new cases of lung cancer and 1.8 million deaths in global, while in China the number was 0.7 million and 0.6 million respectively[2]. Due to the rapidly increasing cases, it is urgent to figure out effective scientific methods to prevent the risk of lung cancer, improving patients' treatment and prolonging the survival time of patients. The tumorigenesis and development of lung cancer is related to behavioral, environmental and genetic discrepancies, among which cigarette smoking is the primary risk factor[3]. Nevertheless, $10 \%-25 \%$ of lung cancer patients are nonsmokers in the world, which shows that the individual genetic factor also plays an important role in carcinogenesis of lung cancer[4].

With the emergence of molecular epidemiology, more and more attention is focusing on common RNA species: non-coding RNAs (ncRNA), as most of the human genome nucleotides can be transcribed for primary transcripts of different cells to produce a range of them by ENCODE pilot project[5]. LncRNAs are one of general non-coding RNAs with a length $>200 \mathrm{nt}$, lacking apparent open-reading frames (ORFs)[6]. On the basis of the GENCODE v7 analysis, lncRNAs have the characteristics of weaker evolutionary constraint and lower expressed, compared with coding genes. It also had canonical gene structures similar to mRNA[7]. Moreover, lncRNAs are preferentially enriched in the nucleus of the cells after transcription, which are involved in nuclear epigenetic modifications and transcriptional regulation of repetitive DNA elements[8]. Because of these multiple features, lncRNAs are abundantly expressed and widely related to a diversity of cancers. Especially, the aberrant expression and mutations of lncRNAs are associated with tumorigenesis, metastasis and tumor stage [9]. Hence, we are able to predict that lncRNAs may be linked to carcinogenesis and promotion of lung cancer.

PVT1 was initially discovered in the mid-80s as a breakpoint site in murine plasmacytomas variant translocations[10]. Its locus was also identified as a retroviral integration site for leukemia virus (MLV)-induced T lymphomas of mice and rats and the analogous of Burkitt's lymphomas in human. PVT1, 1716 nt in length, is located in human chromosomal location 8q24.21 and starts about $57 \mathrm{~kb}$ downstream of well-known MYC oncogene[11]. PVT1 is involved in both physiological and pathological processes, in particular, the tumorigenesis in multifarious cancer, presenting the aberrant level of PVT1 in proliferation, angiogenesis and metastasis of human malignancy[12, 13]. Although the detailed mechanism of PVT1 remains incomplete, decades of study have identified that some molecular functions may influence carcinogenesis by three main pathways. First, intron and exon of PVT1 exhibit the ability to promote tumorigenesis because it participates in interfering with the regulation of multiple oncogenes and anti-oncogenes by DNA rearrangements[14, 15]. Second, the PVT1 locus encodes a range of non-coding RNAs as well as generates a cluster of six annotated microRNAs (namely, miR-1204, miR-1205, miR-126, miR-1207-5p, miR-1207-3p and miR-1208) which can aberrant express in cancer or act as oncogenes or tumor suppressors[16]. Third, PVT1, an activator of oncogene MYC transcription[16], regulates MYC in several different kinds of way. Another study showed that PVT1 affects the protein stability of MYC by Annexin-A2(ANXA2), a kind of Ca2+-binding protein, which binds directly to both ribonucleotide homopolymers and MYC RNA to promote cell proliferation in cancer $[17,18]$. Simultaneously, several studies had shown that PVT1 is upregulated in various human tumors including gastric cancer [19], hepatic carcinoma [20], and prostate cancer[21]. Therefore, we pay our attention to investigating whether PVT1 polymorphisms may be involved in the risk of lung cancer.

Using high-throughput technologies, genomewide association studies (GWAS) have shown evidence of more than one-third of single nucleotide polymorphisms (SNPs) lying within the lncRNAs[22], which may affect gene expression and function and consequently augment the risk of cancer[23, 24]. Accumulating study demonstrated that PVT1 polymorphisms were associated with the risk of various kinds of malignant tumors such as Hodgkin lymphoma[25], pancreatic cancer[26], and follicular lymphoma[27]. Moreover, the studies on the association between the SNPs in lncRNAs and the susceptibility of lung cancer were few so far. Thus we perform a hospital-based case-control study consisting of 515 lung cancer cases and 582 cancer-free controls to evaluate the relevance of the three SNPs and susceptibility of lung cancer.

\section{Materials and Methods}

\section{Study subjects and data collection}

Our research, a hospital-based case-control study, was carried out in Shenyang city which is situated in Liaoning province at the northeast of China. Based on the strict inclusion and exclusion criteria, 515 patients diagnosed with lung cancer (from January 2014 to January 2017) were included in 
the study. The criteria were as follows: (a) patients were newly diagnosed with lung cancer or examined through histopathological examination; (b) patients with no previous cancer or metastatic cancer; (c) patients with no radiotherapy and chemotherapy before diagnosed. Likewise, 582 subjects were enrolled in control group from medical examination centers of the same hospital, matched to case group on age ( \pm 5 years).Subjects devoted $5 \mathrm{ml}$ of venous blood and adopted unified questionnaire to gain the baseline characteristics of all individuals, including gender, age, smoking exposure and so on. Each participant was unrelated ethnic Han Chinese and had signed the informed consent form. Additionally, subjects who have smoked more than 100 cigarettes in their lifetime are identified as smokers while others are defined as non-smokers. The study was approved by the Institutional Review Board of the China Medical University.

\section{SNP selection and genotyping}

By gathering the data of Han Chinese from the 1000 Genome Projects, we gain the genomic information about PVT1 gene, such as genomic sequence, genomic regions, etc. We utilized the Haploview 4.2 software with unified standard to detect the tagSNPs of PVT1. Combining the previous studies of PVT1 polymorphisms on the risk of cancer, we selected three SNPs (rs2608053, rs1561927, rs13254990) to conduct the study ultimately. TaqMan probe, primers and Master Mix were designed and manufactured by Invitrogen (Applied Biosystems). The Genomic DNA samples were extracted from the peripheral blood of the subjects by Phenolchloroform Method. Before utilization, all reagents were stored at $-20^{\circ} \mathrm{C}$. Furthermore, we selected an Applied Biosystems 7500 FAST Real-Time PCR System (Foster City, CA, USA) to conduct PCR reaction and gain fluorescent signal reading. Genotyping results of the three SNPs were presented on the Allelic Discrimination system of SDS software by labeling FAM and VIC fluorescence intensity. For strict quality control, we randomly selected $10 \%$ of the samples to check information integrity and accuracy and conducted duplicate experiments. All the information and results were in accordance.

\section{Statistical analysis}

Considering that the age variable was a continuous variable and gender and smoking exposure were classified variables, we used Student's t-test and X2 test to verify the difference between cases and controls, respectively. Hardy-Weinberg's equilibrium (HWE) for each SNP in control group was calculated by the goodness-of-fit chi-squared test.
Unconditional logistic regression analyses calculated the odds ratios (ORs) and their 95\% confidence intervals (CIs) to evaluate the associations between SNP of rs2608053, rs1561927, rs13254990 and the risk of lung cancer and NSCLC. Additionally, we redefined subjects with both the protective genotype and nonsmoking exposure as control group by cross-over study, analyzing the gene-environment interaction to induce lung cancer through additive model and multiplicative model. The sample capacity was estimated by using "Quanto" software version 1.2.4 (University of Southern California, Los Angeles, CA). All statistical results were calculated by SPSS 21.0 software (IBM SPSS, Inc. Chicago, IL, USA) and a two-side $\mathrm{P}$ value less than 0.05 was considered as significant.

\section{Results}

\section{Demographic Characteristics}

Our case-control study contained 515 cases of lung cancer and 582 controls from health population, which covers non-small cell lung cancer (NSCLC), 278 adenocarcinoma (AD), 176 squamous cell carcinoma (SQ) and 61 small cell lung cancer (SCLC), respectively. Baseline characteristics of both case and control subjects are summarized in table 1. It clearly presented that there were no statistically significant differences in age and gender. The age of cases ranged from 25 to 85 years(mean \pm SD, $59.63 \pm 10.910$ ); the age of controls ranged from 17 to 86 years (mean \pm $\mathrm{SD}, 58.25 \pm 14.612$ ). However, the smoking exposure was $48.0 \%$ in the patients, $23.7 \%$ in the controls, suggesting that the smoking exposure was a risk factor of lung cancer $(\mathrm{P}<0.001)$. Among control group, the genotype frequency distributions of three SNPs were in accordance with Hardy-Weinberg equilibrium $\left(\mathrm{X}^{2}=0.131, \mathrm{P}=0.72\right.$ for $\mathrm{rs} 2608053 ; \mathrm{X}^{2}=0.559$, $\mathrm{P}=0.455$ for $\mathrm{rs} 1561927 ; \quad \mathrm{X}^{2}=0.003, \mathrm{P}=0.955$ for rs13254990) which indicated that the control we selected can represent the general population properly.

\section{Genotype distribution of PVT1 and lung cancer susceptibility}

The unconditional logistic regression analysis was performed to reveal the association with three lncRNAs polymorphisms and the risk of lung cancer as well as NSCLC in Table 2. All the statistical analysis was adjusted by age, gender and smoking status. There was no significantly statistical significance in all genetic (AG vs GG: OR=1.120, 95\% $\mathrm{CI}=0.862-1.455$, $\mathrm{P}=0.396$; AA vs $\mathrm{GG}: \mathrm{OR}=1.218,95 \% \mathrm{CI}=0.711-2.088$, $\mathrm{P}=0.472 ; \quad$ Dominant model: $\mathrm{OR}=1.132$, 95\% CI $=0.880-1.457, \quad \mathrm{P}=0.333 ; \quad$ Recessive model: 
$\mathrm{OR}=1.161,95 \% \mathrm{CI}=0.686-1.967, \mathrm{P}=0.578$, adjusted by age, gender and smoking status) models for rs 2608053 polymorphism. Similarly, rs1561927 and rs13254990 genetic variation was not significantly statistical significance in all models. Moreover, we did not find the statistically significant relationship between three SNPs and the risk of NSCLC (Table 2). According to pathological types of lung cancer, subgroup analysis was performed by stratification of NCLC into lung adenocarcinoma (AD) and lung squamous cell carcinoma (SQ). To be precise, rs13254990 polymorphisms showed observably results in lung adenocarcinoma. Composed with CC genotypes, the risk of lung adenocarcinoma was significantly increased in TT genotype carriers (adjusted $\mathrm{OR}=2.095$, $95 \% \mathrm{CI}=1.084-4.047, \mathrm{P}=0.028)$. In the recessive model, it also showed a statistically significant difference (TT vs $\mathrm{CT}+\mathrm{CC}$ : adjusted $\mathrm{OR}=2.251,95 \% \mathrm{CI}=1.174-4.318$, $\mathrm{P}=0.015$ ).

In further stratified analysis, the result adjusted by age, gender and smoking exposure was presented in Tables 4, 5 and 6. In age subgroup, neither $\leq 59$ years group nor $>59$ years group had statistical correlations. Nevertheless, it was noteworthy that we discovered a borderline significance in both AG genotype compared with AA genotype and dominant model of the rs1561927 subgroups of age $\leq 59$ years $(\mathrm{P}=0.050)$. Compared with the homozygous $\mathrm{CC}$, the patients with the heterozygous CT had a lower risk of lung cancer in the non-smoking group of rs13254990 (adjusted $\mathrm{OR}=0.673,95 \% \mathrm{CI}=0.472-0.959, \mathrm{P}=0.028$ ). The same result consisted in NSCLC that individuals carrying genotype CT has the lower NSCLC risk by 0.685 -fold compared with the individuals carrying genotype CC (adjusted OR $=0.685$, $95 \% \mathrm{CI}=0.477-0.984, \mathrm{P}=0.040)$. Besides, there was no significant effect on the risk of lung cancer considering all of the gender subgroup.

Table 1. Demographic Characteristics of patients with lung cancer and controls.

\begin{tabular}{llll}
\hline Variables & Cases $(\%)(\mathrm{N}=515)$ & Controls $(\%)(\mathrm{N}=582)$ & P-value \\
\hline $\begin{array}{l}\text { Age } \text {,year } \\
(\text { mean } \pm \text { SD) }\end{array}$ & $59.63 \pm 10.910$ & $58.25 \pm 14.612$ & 0.075 \\
$\leq 59$ & $244(47.4)$ & $255(43.8)$ & 0.237 \\
$>59$ & $271(52.6)$ & $327(56.2)$ & \\
Gender & & & 0.722 \\
Female & $244(47.4)$ & $282(48.5)$ & \\
Male & $271(52.6)$ & $300(51.5)$ & 0.000 \\
Smoking status & & & \\
Ever & $247(48.0)$ & $138(23.7)$ & \\
Never & $268(52.0)$ & $444(76.3)$ & \\
Clinical stage & & & \\
I,II & $128(24.9)$ & & \\
III & $185(35.9)$ & & \\
IV & $87(16.9)$ & & \\
Other & $115(22.3)$ & & \\
Pathological type & & & \\
AD & $278(54.0)$ & & \\
SQ & $176(34.2)$ & & \\
SCLC & $61(11.8)$ & & \\
\hline
\end{tabular}

Abbreviations: AD: lung adenocarcinoma; SQ: lung squamous cell carcinoma; SCLC: small-cell lung cancer.

Table 2. Association between the three SNPs and risk of lung cancer and non-small cell lung cancer.

\begin{tabular}{|c|c|c|c|c|c|c|c|}
\hline \multirow[t]{2}{*}{ Genotype } & \multirow{2}{*}{$\begin{array}{l}\text { Controls } \\
(\%)(582)\end{array}$} & \multicolumn{3}{|l|}{ Lung cancer } & \multicolumn{3}{|c|}{ Non-small cell lung cancer } \\
\hline & & Case $(\%)(515)$ & $\mathrm{OR}^{\mathrm{a}}(95 \% \mathrm{CI})$ & $\mathrm{Pb}$-value & Cases (\%)(454) & $\mathrm{OR}^{\mathrm{a}}(95 \% \mathrm{CI})$ & $\mathrm{Pb}$-value \\
\hline \multicolumn{8}{|l|}{ Rs2608053 } \\
\hline GG (ref) & $328(56.4)$ & $283(55.0)$ & 1.00 (ref) & & $242(53.3)$ & 1.00 (ref) & \\
\hline AG & $220(37.8)$ & $202(39.2)$ & $1.120(0.862-1.455)$ & 0.396 & $185(40.7)$ & $1.181(0.903-1.545)$ & 0.224 \\
\hline AA & $34(5.8)$ & $30(5.8)$ & $1.218(0.711-2.088)$ & 0.472 & $27(5.9)$ & $1.256(0,723-2.183)$ & 0.419 \\
\hline $\mathrm{AG}+\mathrm{AA}$ vs $\mathrm{GG}$ & & & $1.132(0.880-1.457)$ & 0.333 & & $1.191(0.919-1.542)$ & 0.186 \\
\hline AA vs $A G+G G$ & & & $1.161(0.686-1.967)$ & 0.578 & & $1.169(0.681-2.006)$ & 0.571 \\
\hline \multicolumn{8}{|l|}{ Rs1561927 } \\
\hline AA (ref) & $547(94.0)$ & $479(93.0)$ & 1.00 (ref) & & $421(92.7)$ & 1.00 (ref) & \\
\hline $\mathrm{AG}$ & $35(6.0)$ & $35(6.8)$ & $1.302(0.786-2.159)$ & 0.306 & $33(7.3)$ & $1.347(0.808-2.247)$ & 0.253 \\
\hline GG & $0(0.0)$ & $1(0.2)$ & - & - & $0(0.0)$ & - & - \\
\hline $\mathrm{AG}+\mathrm{GG}$ vs AA & & & $1.329(0.804-2.196)$ & 0.267 & & $1.347(0.808-2.247)$ & 0.253 \\
\hline GG vs $A G+A A$ & & & - & - & & - & - \\
\hline \multicolumn{8}{|l|}{ Rs13254990 } \\
\hline CC (ref) & $383(65.8)$ & $343(66.6)$ & 1.00 (ref) & & $303(66.7)$ & 1.00 (ref) & \\
\hline $\mathrm{CT}$ & $178(30.6)$ & $144(28.0)$ & $0.842(0.638-1.111)$ & 0.225 & $126(27.8)$ & $0.848(0.637-1.128)$ & 0.257 \\
\hline $\mathrm{TT}$ & $21(3.6)$ & $28(5.4)$ & $1.451(0.776-2.714)$ & 0.243 & $25(5.5)$ & $1.496(0.791-2.830)$ & 0.215 \\
\hline $\mathrm{CT}+\mathrm{TT}$ vs $\mathrm{CC}$ & & & $0.903(0.693-1.176)$ & 0.449 & & $0.913(0.695-1.198)$ & 0.510 \\
\hline $\mathrm{TT}$ vs $\mathrm{CT}+\mathrm{CC}$ & & & $1.529(0.823-2.842)$ & 0.179 & & $1.574(0.837-2.958)$ & 0.159 \\
\hline
\end{tabular}

Abbreviations: SNP: single nucleotide polymorphism; OR: odds ratio; CI: confident interval. Notes: OR ${ }^{\text {a }}$ was adjusted by age, gender and smoking status; $\mathrm{P}_{-}$-value was adjusted by age, gender and smoking status. 
Table 3. Association between the three SNPs and risk of lung adenocarcinoma and squamous cell lung cancer.

\begin{tabular}{|c|c|c|c|c|c|c|c|}
\hline \multirow[t]{2}{*}{ Genotype } & \multirow{2}{*}{$\begin{array}{l}\text { Controls } \\
(\%)(582)\end{array}$} & \multicolumn{3}{|c|}{ Lung adenocarcinoma } & \multicolumn{3}{|c|}{ Lung squamous cell carcinoma } \\
\hline & & Case $(\%)(278)$ & $\mathrm{OR}^{\mathrm{a}}(95 \% \mathrm{CI})$ & $\mathrm{Pb}$-value & Cases (\%)(176) & $\mathrm{OR}^{\mathrm{a}}(95 \% \mathrm{CI})$ & $\mathrm{Pb}$-value \\
\hline \multicolumn{8}{|l|}{ Rs2608053 } \\
\hline GG (ref) & $328(56.4)$ & $147(52.9)$ & 1.00 (ref) & & $95(54.0)$ & 1.00 (ref) & \\
\hline AG & $220(37.8)$ & 111(39.9) & $1.131(0.832-1.538)$ & 0.432 & $74(42.0)$ & $1.270(0.870-1.856)$ & 0.216 \\
\hline AA & $34(5.8)$ & $20(7.2)$ & $1.382(0.758-2.517)$ & 0.291 & $7(4.0)$ & $0.996(0.405-2.446)$ & 0.993 \\
\hline $\mathrm{AG}+\mathrm{AA}$ vs $\mathrm{GG}$ & & & $1.163(0.867-1.562)$ & 0.313 & & $1.239(0.857-1.790)$ & 0.254 \\
\hline AA vs $A G+G G$ & & & $1.311(0.730-2.353)$ & 0.364 & & $0.900(0.372-2.176)$ & 0.815 \\
\hline \multicolumn{8}{|l|}{ Rs1561927 } \\
\hline AA (ref) & $547(94.0)$ & 252(90.6) & & & $169(96.0)$ & & \\
\hline AG & $35(6.0)$ & $26(9.4)$ & $1.637(0.950-2.820)$ & 0.076 & $7(4.0)$ & $0.759(0.317-1.813)$ & 0.534 \\
\hline GG & $0(0.0)$ & $0(0.0)$ & - & - & $0(0.0)$ & - & - \\
\hline AG+GG vs AA & & & $1.637(0.950-2.820)$ & 0.076 & & $0.759(0.317-1.813)$ & 0.534 \\
\hline GG vs AG+AA & & & - & - & & - & - \\
\hline \multicolumn{8}{|l|}{ Rs13254990 } \\
\hline CC (ref) & $383(65.8)$ & $187(67.3)$ & 1.00 (ref) & & $116(65.9)$ & 1.00 (ref) & \\
\hline $\mathrm{CT}$ & 178(30.6) & $70(25.2)$ & $0.782(0.560-1.093)$ & 0.150 & $56(31.8)$ & $1.030(0.694-1.529)$ & 0.883 \\
\hline $\mathrm{TT}$ & $21(3.6)$ & $21(7.6)$ & $2.095(1.084-4.047)$ & $0.028^{*}$ & $4(2.3)$ & $0.570(0.180-1.806)$ & 0.339 \\
\hline $\mathrm{CT}+\mathrm{TT}$ vs $\mathrm{CC}$ & & & $0.913(0.669-1.246)$ & 0.565 & & $0.978(0.666-1.436)$ & 0.911 \\
\hline TT vs CT+CC & & & $2.251(1.174-4.318)$ & $0.015^{*}$ & & $0.564(0.179-1.777)$ & 0.328 \\
\hline
\end{tabular}

Abbreviations: SNP: single nucleotide polymorphism; OR: odds ratio; CI: confident interval; * indicates statistical significance $(P<0.05)$. Notes: OR ${ }^{2}$ was adjusted by age, gender and smoking status; $\mathrm{P}^{\mathrm{b}}$-value was adjusted by age, gender and smoking status.

Table 4. Stratified analyses of association between the three SNPs and lung cancer risk by average age.

\begin{tabular}{|c|c|c|c|c|c|c|c|c|}
\hline \multirow[t]{2}{*}{ Genotype } & \multirow[t]{2}{*}{ Age } & \multirow{2}{*}{$\begin{array}{l}\text { Controls } \\
(\%)(582)\end{array}$} & \multicolumn{3}{|l|}{ Lung cancer } & \multicolumn{3}{|c|}{ Non-small cell lung cancer } \\
\hline & & & Case(\%)(515) & ORa(95\%CI) & $\mathrm{Pb}$-value & Cases(\%)(454) & $\mathrm{ORa}(95 \% \mathrm{CI})$ & $\mathrm{Pb}$-value \\
\hline \multicolumn{9}{|l|}{ Rs2608053 } \\
\hline GG & $\leq 59$ years & $143(56.1)$ & $139(57.0)$ & 1.00 (ref) & & $108(54.3)$ & 1.00 (ref) & \\
\hline AG & & $97(38.0)$ & $92(37.7)$ & $0.832(0.555-1.249)$ & 0.375 & $80(40.2)$ & $0.889(0.581-1.361)$ & 0.589 \\
\hline AA & & $15(5.9)$ & $13(5.3)$ & $0.938(0.403-2.186)$ & 0.882 & $11(5.5)$ & $0.964(0.398-2.333)$ & 0.935 \\
\hline $\mathrm{AG}+\mathrm{AA}$ vs $\mathrm{GG}$ & & & & $0.845(0.572-1.248)$ & 0.398 & & $0.899(0.597-1.353)$ & 0.608 \\
\hline AA vs $A G+G G$ & & & & $1.011(0.442-2.316)$ & 0.979 & & $1.013(0.426-2.405)$ & 0.977 \\
\hline GG & $>59$ years & $185(56.6)$ & 144(53.1) & 1.00 (ref) & & $134(52.5)$ & 1.00 (ref) & \\
\hline AG & & $123(37.6)$ & $110(40.6)$ & $1.354(0.943-1.945)$ & 0.101 & $105(41.2)$ & $1.388(0.962-2.002)$ & 0.080 \\
\hline AA & & $19(5.8)$ & $17(6.3)$ & $1.545(0.748-3.194)$ & 0.240 & $16(6.3)$ & $1.580(0.758-3.296)$ & 0.222 \\
\hline $\mathrm{AG}+\mathrm{AA}$ vs $\mathrm{GG}$ & & & & $1.378(0.973-1.953)$ & 0.071 & & $1.412(0.992-2.009)$ & 0.055 \\
\hline AA vs $A G+G G$ & & & & $1.355(0.668-2.747)$ & 0.400 & & $1.369(0.669-2.799)$ & 0.389 \\
\hline \multicolumn{9}{|l|}{ Rs1561927 } \\
\hline AA & $\leq 59$ years & $244(95.7)$ & $227(93.0)$ & 1.00 (ref) & & 183(92.0) & 1.00 (ref) & \\
\hline AG & & $11(4.3)$ & $16(6.6)$ & $1.920(0.814-4.530)$ & 0.136 & $16(8.0)$ & $2.369(1.002-5.604)$ & $0.050^{*}$ \\
\hline GG & & $0(0.0)$ & $1(0.4)$ & - & - & $0(0.0)$ & - & - \\
\hline AG+GG vs AA & & & & $1.998(0.855-4.669)$ & 0.110 & & $2.369(1.002-5.604)$ & 0.050 * \\
\hline GG vs AG+AA & & & & - & - & & - & - \\
\hline AA & $>59$ years & $303(92.7)$ & 255(93.0) & 1.00 (ref) & & 238(93.3) & 1.00 (ref) & \\
\hline AG & & $24(7.3)$ & $19(7.0)$ & $1.252(0.649-2.417)$ & 0.503 & $17(6.7)$ & $1.144(0.581-2.251)$ & 0.697 \\
\hline GG & & $0(0.0)$ & $0(0.0)$ & - & - & $0(0.0)$ & - & - \\
\hline $\mathrm{AG}+\mathrm{GG}$ vs $\mathrm{AA}$ & & & & $1.252(0.649-2.417)$ & 0.503 & & $1.144(0.581-2.251)$ & 0.697 \\
\hline GG vs AG+AA & & & & - & - & & - & - \\
\hline \multicolumn{9}{|l|}{ Rs13254990 } \\
\hline $\mathrm{CC}$ & $\leq 59$ years & $166(65.1)$ & $166(68.0)$ & 1.00 (ref) & & $134(67.3)$ & 1.00 (ref) & \\
\hline CT & & $80(31.4)$ & $64(26.2)$ & $0.753(0.490-1.159)$ & 0.197 & $54(27.1)$ & $0.790(0.503-1.241)$ & 0.306 \\
\hline TT & & $9(3.5)$ & $14(5.7)$ & $1.699(0.646-4.467)$ & 0.282 & $11(5.5)$ & $1.795(0.654-4.927)$ & 0.256 \\
\hline $\mathrm{CT}+\mathrm{TT}$ vs $\mathrm{CC}$ & & & & $0.840(0.558-1.263)$ & 0.402 & & $0.879(0.572-1.350)$ & 0.555 \\
\hline TT vs $\mathrm{CT}+\mathrm{CC}$ & & & & $1.849(0.710-4.815)$ & 0.208 & & $1.928(0.709-5.240)$ & 0.198 \\
\hline $\mathrm{CC}$ & $>59$ years & $217(66.4)$ & 177(65.3) & 1.00 (ref) & & $169(66.3)$ & 1.00 (ref) & \\
\hline $\mathrm{CT}$ & & $98(30.0)$ & $80(29.5)$ & $0.958(0.657-1.398)$ & 0.826 & $72(28.2)$ & $0.913(0.621-1.341)$ & 0.641 \\
\hline $\mathrm{TT}$ & & $12(3.7)$ & $14(5.2)$ & $1.281(0.546-3.003)$ & 0.569 & $14(5.5)$ & $1.335(0.572-3.117)$ & 0.504 \\
\hline $\mathrm{CT}+\mathrm{TT}$ vs CC & & & & $0.994(0.693-1.426)$ & 0.975 & & $0.960(0.666-1.384)$ & 0.826 \\
\hline TT vs $\mathrm{CT}+\mathrm{CC}$ & & & & $1.298(0.558-3.018)$ & 0.545 & & $1.373(0.593-3.179)$ & 0.460 \\
\hline
\end{tabular}

Abbreviations: SNP: single nucleotide polymorphism; OR: odds ratio; CI: confident interval; * indicates statistical significance $(P<0.05)$. Notes: ORa was adjusted by age, gender and smoking status; $\mathrm{P}^{\mathrm{b}}$-value was adjusted by age, gender and smoking status. 


\section{Interaction results between SNPs and smoking status}

To investigate the additive interaction of three SNPs and smoking status on lung cancer susceptibility, we implemented a crossover analysis and showed the measure results in Table 7 and 8. For rs1561927, those carriers of AG and GG genotype with smoking exposure had a higher lung cancer risk by 7.108-fold and a higher NSCLC risk by 6.856-foldcompared with the carriers of AA with no smoking exposure (adjusted OR=7.108, 95\% $\mathrm{CI}=2.363-21.376, \quad \mathrm{P}=0.000 ;$ adjusted $\mathrm{OR}=6.856$, 95\% $\mathrm{CI}=2.237-21.017, \mathrm{P}=0.001$ ). For rs2608053, the participants with both AG/AA genotype and smoking were possible to induce lung cancer and NSCLC compared with GG genotype with non-smoking exposure (adjusted OR=5.849, 95\% $\mathrm{CI}=3.691-9.269, \quad \mathrm{P}=0.000 ; \quad$ adjusted $\mathrm{OR}=5.472$, $95 \% \mathrm{CI}=3.418-8.762, \mathrm{P}=0.000)$. Similar results were also showed in rs13254990 polymorphism. However, three measuring results of rs2608053, rs1561927 and rs13254990 were not statistically significant, which indicated that there was no additive interaction between gene-smoking factor of rs2608053, rs1561927 and rs13254990. In multiplicative interaction analysis, the result showed that there were no statistically meaningful gene-smoking interactions (data were not shown).

In order to further investigate the association of PVT1 with a variety of cancer development, we summarized the effects of PVT1 inhibiting on malignant phenotypes (including proliferation, metastasis, invasion, and apoptosis) and its diverse molecular mechanisms (see Additional file 1: Table S1)[21, 28-70]. Additionally, PVT1 possesses multifarious molecular functions, including transcriptional dysregulation, pre-mRNA alternative splicing, ceRNA role, epigenetic alterations and transition of cell phenotype through different signaling pathways covering EMT and other pathways. To generalize, PVT1 might exert its molecular function via regulating the expression of related genes and miRNA, affecting carcinogenic signaling pathways, which might induce malignant carcinoma. Moreover, up-regulation of PVT1, comparing with down-regulation of PVT1, might have poor prognosis in various cancers.

Table 5. Association of the three SNPs with lung cancer risks and non-small-cell lung cancer risks in female and male populations.

\begin{tabular}{|c|c|c|c|c|c|c|c|c|}
\hline \multirow[t]{2}{*}{ Genotype } & \multirow[t]{2}{*}{ Gender } & \multirow{2}{*}{$\begin{array}{l}\text { Controls } \\
(\%)(582)\end{array}$} & \multicolumn{3}{|l|}{ Lung cancer } & \multicolumn{3}{|c|}{ Non-small cell lung cancer } \\
\hline & & & Case(\%)(515) & ORa $(95 \% \mathrm{CI})$ & $\mathrm{Pb}$-value & Cases $(\%)(454)$ & $\mathrm{OR} \mathrm{a}(95 \% \mathrm{CI})$ & $\mathrm{Pb}$-value \\
\hline \multicolumn{9}{|l|}{ Rs2608053 } \\
\hline GG & Female & $151(53.5)$ & $121(49.6)$ & 1.00 (ref) & & $108(48.2)$ & 1.00 (ref) & \\
\hline AG & & $111(39.4)$ & 107(43.9) & $1.249(0.862-1.809)$ & 0.240 & $101(45.1)$ & $1.339(0.916-1.958)$ & 0.131 \\
\hline AA & & $20(7.1)$ & $16(6.6)$ & $1.169(0.577-2.368)$ & 0.664 & $15(6.7)$ & $1.246(0.607-2.559)$ & 0.549 \\
\hline $\mathrm{AG}+\mathrm{AA}$ vs $\mathrm{GG}$ & & & & $1.237(0.867-1.765)$ & 0.241 & & $1.325(0.920-1.909)$ & 0.130 \\
\hline AA vs AG+GG & & & & $1.058(0.533-2.098)$ & 0.873 & & $1.089(0.542-2.187)$ & 0.811 \\
\hline GG & Male & $177(59.0)$ & $162(59.8)$ & 1.00 (ref) & & $134(58.3)$ & 1.00 (ref) & \\
\hline AG & & $109(36.3)$ & $95(35.1)$ & $0.986(0.677-1.436)$ & 0.941 & $84(36.5)$ & $1.034(0.701-1.524)$ & 0.868 \\
\hline AA & & $14(4.7)$ & $14(5.2)$ & $1.304(0.562-3.026)$ & 0.536 & $12(5.2)$ & $1.312(0.549-3.132)$ & 0.541 \\
\hline $\mathrm{AG}+\mathrm{AA}$ vs $\mathrm{GG}$ & & & & $1.019(0.709-1.463)$ & 0.920 & & $1.062(0.730-1.544)$ & 0.752 \\
\hline AA vs AG+GG & & & & $1.311(0.572-3.005)$ & 0.522 & & $1.295(0.550-3.049)$ & 0.555 \\
\hline \multicolumn{9}{|l|}{ Rs1561927 } \\
\hline AA & Female & $261(92.6)$ & $223(91.4)$ & 1.00 (ref) & & 205(91.5) & 1.00 (ref) & \\
\hline AG & & $21(7.4)$ & $21(8.6)$ & $1.334(0.702-2.536)$ & 0.379 & $19(8.5)$ & $1.294(0.670-2.500)$ & 0.443 \\
\hline GG & & $0(0.0)$ & $0(0.0)$ & - & - & $0(0.0)$ & - & - \\
\hline $\mathrm{AG}+\mathrm{GG}$ vs $\mathrm{AA}$ & & & & $1.334(0.702-2.536)$ & 0.379 & & $1.294(0.670-2.500)$ & 0.443 \\
\hline GG vs $A G+A A$ & & & & - & - & & - & - \\
\hline AA & Male & $286(95.3)$ & $256(94.5)$ & 1.00 (ref) & & $216(93.9)$ & 1.00 (ref) & \\
\hline AG & & $14(4.7)$ & $14(5.2)$ & $1.381(0.608-3.319)$ & 0.441 & $14(6.1)$ & $1.585(0.700-3.588)$ & 0.269 \\
\hline GG & & $0(0.0)$ & $1(0.4)$ & - & - & $0(0.0)$ & - & - \\
\hline$A G+G G$ vs $A A$ & & & & $1.460(0.651-3.274)$ & 0.358 & & $1.585(0.700-3.588)$ & 0.269 \\
\hline GG vs AG+AA & & & & - & - & & - & - \\
\hline \multicolumn{9}{|l|}{ Rs13254990 } \\
\hline $\mathrm{CC}$ & Female & $183(64.9)$ & $168(68.9)$ & 1.00 (ref) & & $155(69.2)$ & 1.00 (ref) & \\
\hline $\mathrm{CT}$ & & $91(32.3)$ & $64(26.2)$ & $0.687(0.460-1.025)$ & 0.066 & $59(26.3)$ & $0.691(0.458-1.042)$ & 0.078 \\
\hline TT & & $8(2.8)$ & $12(4.9)$ & $1.223(0.455-3.289)$ & 0.689 & $10(4.5)$ & $1.176(0.423-3.265)$ & 0.756 \\
\hline $\mathrm{CT}+\mathrm{TT}$ vs $\mathrm{CC}$ & & & & $0.730(0.497-1.073)$ & 0.109 & & $0.731(0.493-1.083)$ & 0.118 \\
\hline TT vs $\mathrm{CT}+\mathrm{CC}$ & & & & $1.369(0.513-3.650)$ & 0.530 & & $1.313(0.476-3.617)$ & 0.599 \\
\hline $\mathrm{CC}$ & Male & $200(66.7)$ & $175(64.6)$ & 1.00 (ref) & & $148(64.3)$ & 1.00 (ref) & \\
\hline $\mathrm{CT}$ & & $87(29.0)$ & $80(29.5)$ & $1.011(0.681-1.499)$ & 0.958 & $67(29.1)$ & $1.025(0.680-1.546)$ & 0.905 \\
\hline TT & & $13(4.3)$ & $16(5.9)$ & $1.541(0.673-3.527)$ & 0.306 & $15(6.5)$ & $1.708(0.741-3.940)$ & 0.209 \\
\hline $\mathrm{CT}+\mathrm{TT}$ vs $\mathrm{CC}$ & & & & $1.075(0.740-1.561)$ & 0.706 & & $1.109(0.752-1.634)$ & 0.602 \\
\hline TT vs $\mathrm{CT}+\mathrm{CC}$ & & & & $1.536(0.677-3.485)$ & 0.304 & & $1.695(0.742-3.872)$ & 0.211 \\
\hline
\end{tabular}

Abbreviations: SNP: single nucleotide polymorphism; OR: odds ratio; CI: confident interval; * indicates statistical significance $(P<0.05)$. Notes: ORa was adjusted by age, gender and smoking status; $\mathrm{P}^{\mathrm{b}}$-value was adjusted by age, gender and smoking status. 
Table 6. Stratified analyses of the three SNPs with lung cancer risks and non-small-cell lung cancer risks by smoking status.

\begin{tabular}{|c|c|c|c|c|c|c|c|c|}
\hline \multirow[t]{2}{*}{ Genotype } & \multirow{2}{*}{$\begin{array}{l}\text { Smoking } \\
\text { exposure }\end{array}$} & \multirow{2}{*}{$\begin{array}{l}\text { Controls } \\
(\%)(582)\end{array}$} & \multicolumn{3}{|l|}{ Lung cancer } & \multicolumn{3}{|c|}{ Non-small cell lung cancer } \\
\hline & & & Case(\%)(515) & $\mathrm{ORa}(95 \% \mathrm{CI})$ & $\mathrm{Pb}$-value & Cases(\%)(454) & $\mathrm{OR} a(95 \% \mathrm{CI})$ & $\mathrm{Pb}$-value \\
\hline \multicolumn{9}{|l|}{ Rs2608053 } \\
\hline GG & Ever & $83(60.1)$ & $152(61.5)$ & 1.00 (ref) & & $123(59.7)$ & 1.00 (ref) & \\
\hline AG & & $52(37.7)$ & $84(34.0)$ & $0.894(0.569-1.405)$ & 0.628 & $74(35.9)$ & $1.026(0.639-1.646)$ & 0.917 \\
\hline AA & & $3(2.2)$ & $11(4.5)$ & $2.177(0.586-8.083)$ & 0.245 & $9(4.4)$ & $2.243(0.581-8.660)$ & 0.241 \\
\hline $\mathrm{AG}+\mathrm{AA}$ vs $\mathrm{GG}$ & & & & $0.966(0.623-1.500)$ & 0.879 & & $1.096(0.692-1.737)$ & 0.696 \\
\hline AA vs $A G+G G$ & & & & $2.267(0.617-8.332)$ & 0.218 & & $2.222(0.582-8.477)$ & 0.243 \\
\hline GG & Never & $245(55.2)$ & $131(48.9)$ & 1.00 (ref) & & & 1.00 (ref) & \\
\hline AG & & $168(37.8)$ & $118(44.0)$ & $1.279(0.927-1.764)$ & 0.135 & $119(48.0)$ & $1.314(0.946-1.827)$ & 0.104 \\
\hline AA & & $31(7.0)$ & $19(7.1)$ & $1.109(0.598-2.059)$ & 0.742 & $111(44.7)$ & $1.153(0.615-2.162)$ & 0.657 \\
\hline $\mathrm{AG}+\mathrm{AA}$ vs $\mathrm{GG}$ & & & & $1.252(0.920-1.705)$ & 0.153 & $18(7.3)$ & $1.289(0.940-1.768)$ & 0.115 \\
\hline AA vs $A G+G G$ & & & & $0.995(0.546-1.815)$ & 0.987 & & $1.020(0.554-1.879)$ & 0.949 \\
\hline \multicolumn{9}{|l|}{ Rs1561927 } \\
\hline AA & Ever & $133(96.4)$ & $236(95.5)$ & 1.00 (ref) & & 196(95.1) & 1.00 (ref) & \\
\hline AG & & $5(3.6)$ & $10(4.0)$ & $1.255(0.406-3.876)$ & 0.693 & $10(4.9)$ & $1.609(0.514-5.041)$ & 0.414 \\
\hline GG & & $0(0.0)$ & $1(0.4)$ & - & - & $0(0.0)$ & - & - \\
\hline$A G+G G$ vs AA & & & & $1.399(0.462-4.238)$ & 0.552 & & $1.609(0.514-5.041)$ & 0.414 \\
\hline GG vs AG+AA & & & & - & - & & - & - \\
\hline AA & Never & $414(93.2)$ & $243(90.7)$ & 1.00 (ref) & & $225(90.7)$ & 1.00 (ref) & \\
\hline AG & & $30(6.8)$ & $25(9.3)$ & $1.383(0.787-2.429)$ & 0.260 & $23(9.3)$ & $1.367(0.769-2.430)$ & 0.287 \\
\hline GG & & $0(0.0)$ & $0(0.0)$ & - & - & $0(0.0)$ & - & - \\
\hline $\mathrm{AG}+\mathrm{GG}$ vs AA & & & & $1.383(0.787-2.429)$ & 0.260 & & $1.367(0.769-2.430)$ & 0.287 \\
\hline GG vs AG+AA & & & & - & - & & - & - \\
\hline \multicolumn{9}{|l|}{ Rs13254990 } \\
\hline $\mathrm{CC}$ & Ever & $93(67.4)$ & $151(61.1)$ & 1.00 (ref) & & $126(61.2)$ & 1.00(ref) & \\
\hline $\mathrm{CT}$ & & $39(28.3)$ & $81(32.8)$ & $1.252(0.777-2.016)$ & 0.355 & $67(32.5)$ & $1.273(0.772-2.099)$ & 0.345 \\
\hline TT & & $6(4.3)$ & $15(6.1)$ & $1.304(0.471-3.611)$ & 0.609 & $13(6.3)$ & $1.433(0.502-4.086)$ & 0.501 \\
\hline $\mathrm{CT}+\mathrm{TT}$ vs CC & & & & $1.259(0.800-1.981)$ & 0.319 & & $1.295(0.805-2.082)$ & 0.286 \\
\hline $\mathrm{TT}$ vs $\mathrm{CT}+\mathrm{CC}$ & & & & $1.214(0.443-3.323)$ & 0.707 & & $1.327(0.471-3.740)$ & 0.592 \\
\hline $\mathrm{CC}$ & Never & $290(65.3)$ & 192(71.6) & 1.00 (ref) & & $177(71.4)$ & 1.00 (ref) & \\
\hline $\mathrm{CT}$ & & $139(31.3)$ & $63(23.5)$ & $0.673(0.472-0.959)$ & $0.028^{*}$ & $59(23.8)$ & $0.685(0.477-0.984)$ & $0.040^{*}$ \\
\hline TT & & $15(3.4)$ & $13(4.9)$ & $1.465(0.670-3.206)$ & 0.339 & $12(4.8)$ & $1.471(0.662-3.268)$ & 0.344 \\
\hline $\mathrm{CT}+\mathrm{TT}$ vs $\mathrm{CC}$ & & & & $0.744(0.532-1.039)$ & 0.083 & & $0.755(0.536-1.063)$ & 0.107 \\
\hline $\mathrm{TT}$ vs $\mathrm{CT}+\mathrm{CC}$ & & & & $1.639(0.754-3.563)$ & 0.213 & & $1.638(0.742-3.615)$ & 0.222 \\
\hline
\end{tabular}

Abbreviations: SNP: single nucleotide polymorphism; OR: odds ratio; CI: confident interval; * indicates statistical significance $(P<0.05)$. Notes: ORa was adjusted by age and gender; $\mathrm{P}^{\mathrm{b}}$-value was adjusted by age, gender and smoking status.

Table 7. Crossover analysis of interaction between rs2608053, rs 1561927 and rs 13254990 risk genotypes and smoking exposure.

\begin{tabular}{|c|c|c|c|c|c|c|c|c|c|}
\hline \multirow[t]{2}{*}{ SNPs } & \multirow[t]{2}{*}{ Genotype } & \multirow{2}{*}{$\begin{array}{l}\text { Smoking } \\
\text { exposure }\end{array}$} & \multirow{2}{*}{$\begin{array}{l}\text { Controls } \\
(\%)(582)\end{array}$} & \multicolumn{3}{|l|}{ Lung cancer } & \multicolumn{3}{|c|}{ Non-small cell lung cancer } \\
\hline & & & & Cases(\%)(515) & ORa(95\%CI) & Pb-value & Cases $(\%)(454)$ & $\mathrm{ORa}(95 \% \mathrm{CI})$ & $\mathrm{Pb}$-value \\
\hline \multirow[t]{4}{*}{ Rs2608053 } & GG & Never & $245(42.1)$ & $131(25.4)$ & 1.00 (ref) & & $119(26.2)$ & 1.00 (ref) & \\
\hline & $\mathrm{AG}+\mathrm{AA}$ & Never & $199(34.2)$ & $137(26.6)$ & $1.242(0.911-1.694)$ & 0.170 & $129(28.4)$ & $1.275(0.928-1.753)$ & 0.134 \\
\hline & GG & Ever & $83(14.3)$ & $152(29.5)$ & $6.175(4.073-9.364)$ & $0.000^{*}$ & $123(27.1)$ & $5.256(3.429-8.054)$ & $0.000^{*}$ \\
\hline & $\mathrm{AG}+\mathrm{AA}$ & Ever & $55(9.5)$ & 95(18.4) & $5.849(3.691-9.269)$ & $0.000^{*}$ & $83(18.3)$ & $5.472(3.418-8.762)$ & $0.000^{*}$ \\
\hline \multirow[t]{4}{*}{ Rs1561927 } & AA & Never & $414(71.1)$ & $243(47.2)$ & 1.00 (ref) & & $225(49.6)$ & 1.00 (ref) & \\
\hline & $\mathrm{AG}+\mathrm{GG}$ & Never & $30(5.2)$ & $25(4.9)$ & $1.342(0.762-2.363)$ & 0.309 & $23(5.1)$ & $1.326(0.743-2.367)$ & 0.339 \\
\hline & AA & Ever & $133(22.9)$ & $236(45.8)$ & $5.537(3.878-7.904)$ & $0.000^{*}$ & $196(43.2)$ & $4.806(3.343-6.910)$ & $0.000^{*}$ \\
\hline & $\mathrm{AG}+\mathrm{GG}$ & Ever & $5(0.9)$ & $11(2.1)$ & $7.108(2.363-21.376)$ & $0.000^{*}$ & $10(2.2)$ & $6.856(2.237-21.017)$ & $0.001^{*}$ \\
\hline \multirow[t]{4}{*}{ Rs13254990 } & $\mathrm{CC}+\mathrm{CT}$ & Never & $429(73.7)$ & $255(49.5)$ & 1.00 (ref) & & $236(52.0)$ & 1.00 (ref) & \\
\hline & $\mathrm{TT}$ & Never & $15(2.6)$ & $13(2.5)$ & $1.689(0.773-3.692)$ & 0.189 & $12(2.6)$ & $1.697(0.763-3.776)$ & 0.195 \\
\hline & $\mathrm{CC}+\mathrm{CT}$ & Ever & $132(22.7)$ & $232(45.0)$ & $5.555(3.880-7.953)$ & $0.000^{*}$ & $193(42.5)$ & 4.833(3.354-6.966) & $0.000^{*}$ \\
\hline & $\mathrm{TT}$ & Ever & $6(1.0)$ & $15(2.9)$ & $7.238(2.680-19.549)$ & $0.000^{*}$ & $13(2.9)$ & $6.740(2.439-18.626)$ & $0.000^{*}$ \\
\hline
\end{tabular}

Abbreviations: SNP: single nucleotide polymorphism; OR: odds ratio; CI: confident interval; * indicates statistical significance $(P<0.05)$. Notes: ORa was adjusted by age and gender; $\mathrm{P}$-value was adjusted by age and gender.

\section{Discussion}

This is the first study on the association between lncRNA PVT1 polymorphisms and the susceptibility of lung cancer. It is also the first report on geneenvironment interaction between three polymorphisms and smoking status through additive and multiplicative model. The homozygous variant genotype and recessive model of rs13254990 were considered to be related to prominent increase in the risk of lung adenocarcinoma. Additionally, the heterozygote CT genotype of rs13254990 decreased the susceptibility to lung cancer and NSCLC in nonsmokers. Analyzing the measures of additive and multiplicative model, we did not discover the gene-environment interaction. 
Table 8. Addictive interaction between rs2608053, rs1561927, rs13254990 risk genotypes and smoking exposure.

\begin{tabular}{llllll}
\hline SNPs & Measure & \multicolumn{2}{l}{ Lung cancer } & \multicolumn{2}{l}{$\begin{array}{l}\text { Non-small cell lung } \\
\text { cancer }\end{array}$} \\
\cline { 3 - 6 } & & Estimate & $95 \% \mathrm{CI}$ & Estimate & $95 \% \mathrm{CI}$ \\
\hline Rs2608053 & RERI & -0.568 & -3.174 to & -0.059 & -2.481 to \\
& & & 2.038 & & 2.364 \\
& AP & -0.097 & -0.565 to & -0.011 & -0.456 to \\
& & & 0.371 & & 0.435 \\
& S & 0.895 & 0.537 to & 0.987 & 0.576 to \\
Rs1561927 & RERI & -1.272 & -2.441 to & -1.243 & -2.415 to \\
& & & -0.103 & & -0.072 \\
& AP & -0.948 & -2.239 to & -0.937 & -2.247 to \\
& & & 0.343 & & 0.373 \\
& S & 0.212 & 0.021 to & 0.208 & 0.018 to \\
Rs13254990 & RERI & -0.963 & -2.106 & & 2.357 \\
& & & 0.577 to & -0.910 & -2.475 to \\
& AP & -0.570 & -1.871 to & -0.536 & -1.835 to \\
& & & 0.731 & & 0.763 \\
& S & 0.417 & 0.059 to & 0.434 & 0.060 to \\
& & & 2.944 & & 3.153 \\
\hline
\end{tabular}

Abbreviations: CI: confidence interval; RERI: relative excess risk due to interaction; AP: attributable proportion due to interaction; S: synergy index.

LncRNAs were detectable and stabilized in body fluids, with a variety of biologic functions, especially ectopic expression, serving as a regulator of tumorigenesis[71]. To be precise, it may affects the proliferation, invasion, migration, metastasis and apoptosis of tumor cells through regulating part of oncogenes and tumor suppressors both at transcriptional and posttranscriptional levels[72, 73]. Moreover, it may also induce epithelial-to-mesenchymal transition (EMT) by the PI3K-AKT and miR-143/HMGB1 pathway[74, 75] to promote cancer metastasis. Since lncRNA had played an important part in the emergence, development and even therapy and prognosis of multiple cancers, PVT1, as an emerging gene, got more attention. Up to present, PVT1 was reported to play important roles in the development of lung cancer and NSCLC, by some researchers. Li et al. suggested that PVT1-5 expression was significantly increased to lung cancer tissues and cell lines and was stimulated lung cancer progression by PVT1-5/miR-126/SLC7A5 regulatory network. PVT1-5 positively regulates posttranscriptional expression of SLC7A5 by sponging miR-126, resulting in the cell proliferation[70]. Another study revealed that Yin Yang-1(YY1), a multifunctional transcription factor, bound to the promoter region of PVT1 and motivated its transcription through the consensus YY1 motif to promote cell proliferation, migration and invasion in lung cancer[69]. Likewise, the expression of PVT1 was also increased to NSCLC cell lines, thus promoting the invasive ability of NSCLC cells. As a competitive endogenous RNA(ceRNA), PVT1 suppressed MMP9 expression via competitively binding miR-200a and miR-200b to destroy the reconstruction ability of respiratory tract and lung tissues to induce NSCLC, suggesting that the lncRNA-PVT1-MMP9 axis may be a potential target for NCSLC metastasis[76]. Additionally, Wan et al. illustrated that PVT1 could directly bind to EZH2, a core subunit of the PRC2 complex, to repress the large tumor suppressor kinase 2(LAYS2) transcription in A459 and PC-9 cells. Thus, it induced cell proliferation, induces apoptosis, and promotes cell-cycle arrest in NSCLC, especially lung adenocarcinoma[77]. Consequently, we conducted our case-control study and stratified lung cancer into NSCLC, lung adenocarcinoma and lung squamous cell carcinoma to further analysis the association between PVT1 polymorphisms and lung cancer.

Emerging evidence of Genome-wide association study had demonstrated that SNPs located in PVT1 might be used as susceptibility factors to several cancers. Zhang et al. showed that PVT1 affected cell proliferation in breast cancer via increasing the GG genotype of rs13281615[78]. Furthermore, PVT1 polymorphisms were regulated the prognosis of cancer, too. Zhang et al. illustrated that the presence of rs13281615 G>A polymorphism on PVT1 affected a favorable prognosis in colon cancer patients through modulating the activity of the PVT1/miR-146a/COX2 signaling pathway[79]. For SNPs that conducted in our study, Victor et al. conducted a genome-wide association study (GWAS) of 589 classical Hodgkin Lyphoma (cHL) patients and 5,199 free-controls and replication studies to identify predisposition loci of cHL[25], suggesting that rs2608053 might be associated with cHL (OR=1.20, 95\% CI=1.12-1.28, $\left.\mathrm{P}=1.16 \times 10^{-7}\right)$. For rs1561927, it was identified as a risk locus for pancreatic cancer, which located at $455 \mathrm{~kb}$ telomeric of PVT1, a nongenic region between PVT1 and LINC00977[26]. Moschovis et al. performed a case-control study and indicated that PVT1 rs1561927 G allele was significantly overrepresented in both pancreatic ductal adenocarcinoma (PDAC) cases and pancreatic neuroendocrine tumor (PNET) cases[80]. Additionally, Christine et al. revealed Follicular Lymphoma (FL) susceptibility loci rs13254990 by proceeding a large-scale two-stage GWAS in 4,523 patients and 13,344 controls of European ancestry[27]. Nevertheless, there were some limitations about clarification of causal SNPs and the deep mining of GWAS data. According to it was the first study and the limitations, we chose experimental design and implementation to further explore the relationship between lung cancer and PVT1 polymorphisms. Based on the study of molecular mechanism and PVT1 polymorphisms, we performed the case-control study. In accordance with our study, we found that, as susceptibility loci, the polymorphisms of were associated with the risk of lung cancer in several 
stratified analysis. It was demonstrated in the analysis that rs13254990 polymorphism increased the risk of lung adenocarcinoma, but it decreased the risk of lung cancer and NCSLC susceptibility in nonsmokers. This is partly due to insufficient capacity and different smoking status. However, rs2608053 and rs1561927 polymorphisms were not related to the risk of lung cancer, which may due to different ethnicities and the lack of data about validate functional experiments.

Several deficiencies in the present case-control study should be emphatically taken into account. First, all study subjects were enrolled from the three hospitals in northeast China with limited sample size, which could lead to Berkson's bias. Secondly, when collecting demographic data, smoking status offered by individuals may result in recall bias and other environmental risk factors of lung cancer could not be found, so these might confuse the associations between SNP and lung cancer susceptibility. Thirdly, functional verification of three SNPs in PVT1 did not perform in present study. Therefore, the underlying mechanisms need to be elucidated with more large-scale subjects across different ethnicities in the future.

\section{Conclusion}

The polymorphism rs13254990 in PVT1 gene is associated with the risk of lung adenocarcinoma in a Chinese northeast population. The interactions between three polymorphisms and smoking status were not statistically significant.

\section{Abbreviations}

LncRNA: long non-coding RNA; PVT1: plasmacytoma variant translocation 1; SNP: single nucleotide polymorphism; NSCLC: non-small cell lung cancer; AD: lung adenocarcinoma; SQ: lung squamous cell carcinoma; SCLC: small cell lung cancer; HWE: Hardy-Weinberg equilibrium; 95\% CI: 95\% confidence interval; OR: odds ratio; Real-Time PCR: real-time polymerase chain reaction; GWAS: genome-wide association study.

\section{Supplementary Material}

Supplementary figures and tables. http://www.jcancer.org/v11p0468s1.pdf

\section{Acknowledgements}

The authors are grateful to the patients for their participation. They would like to thank all hospital personnel involved in this study.

\section{Ethics approval and informed consent}

The study has acquired approval of the Institutional Review Board of China Medical
University, and all enrolled participants or their representatives signed the informed consent according to relevant regulations. All participants signed informed consent in the study.

\section{Data availability}

The data of the study are available from the corresponding author on reasonable request.

\section{Funding}

This study was supported by the National Natural Science Foundation of China (NO.81673261).

\section{Authors' contributions}

$\mathrm{ZW} \mathrm{Z}$ and $\mathrm{ZH} \mathrm{Y}$ designed experiments and wrote the paper. $\mathrm{H} \mathrm{L}$ and J L collected data. XT L and ZT Y analyzed the data. M G, YH B and SL W interpreted results. ZG C and BS $Z$ edited the paper. All authors have read and approved the final manuscript.

\section{Competing Interests}

The authors have declared that no competing interest exists.

\section{References}

1. Bray F, Ferlay J, Soerjomataram I, Siegel RL, Torre LA, Jemal A. Global cancer statistics 2018: GLOBOCAN estimates of incidence and mortality worldwide for 36 cancers in 185 countries. CA: a cancer journal for clinicians. 2018; 68: 394-424

2. [Internet] Cancer Today http://gco.iarc.fr/today/home.

3. Malhotra J, Malvezzi M, Negri E, La Vecchia C, Boffetta P. Risk factors for lung cancer worldwide. Eur Respir J. 2016; 48: 889-902.

4. Rivera GA, Wakelee H. Lung Cancer in Never Smokers. Adv Exp Med Biol. 2016; 893: 43-57.

5. Birney E, Stamatoyannopoulos JA, Dutta A, Guigo R, Gingeras TR, Margulies $\mathrm{EH}$, et al. Identification and analysis of functional elements in $1 \%$ of the human genome by the ENCODE pilot project. Nature. 2007; 447: 799-816.

6. Ponting $\mathrm{CP}$, Oliver PL, Reik W. Evolution and functions of long noncoding RNAs. Cell. 2009; 136: 629-41.

7. Derrien T, Johnson R, Bussotti G, Tanzer A, Djebali S, Tilgner H, et al. The GENCODE v7 catalog of human long noncoding RNAs: analysis of their gene structure, evolution, and expression. Genome Res. 2012; 22: 1775-89.

8. Chen L, Zhou Y, Li H. LncRNA, miRNA and IncRNA-miRNA interaction in viral infection. Virus Res. 2018; 257: 25-32.

9. Bhan A, Soleimani M, Mandal SS. Long Noncoding RNA and Cancer: A New Paradigm. Cancer Res. 2017; 77: 3965-81.

10. Webb E, Adams JM, Cory S. Variant $(6 ; 15)$ translocation in a murine plasmacytoma occurs near an immunoglobulin kappa gene but far from the myc oncogene. Nature. 1984; 312: 777-9.

11. Shtivelman E, Henglein B, Groitl P, Lipp M, Bishop JM. Identification of a human transcription unit affected by the variant chromosomal translocations 2;8 and 8;22 of Burkitt lymphoma. Proc NatI Acad Sci U S A. 1989; 86: 3257-60.

12. Zheng $X, \mathrm{Hu} \mathrm{H}, \mathrm{Li}$ S. High expression of lncRNA PVT1 promotes invasion by inducing epithelial-to-mesenchymal transition in esophageal cancer. Oncol Lett. 2016; 12: 2357-62.

13. Huang C, Liu S, Wang $H$, Zhang Z, Yang Q, Gao F. LncRNA PVT1 overexpression is a poor prognostic biomarker and regulates migration and invasion in small cell lung cancer. Am J Transl Res. 2016; 8: 5025-34.

14. Tsutsumi Y, Chinen Y, Sakamoto N, Nagoshi H, Nishida K, Kobayashi S, et al. Deletion or methylation of CDKN2A/2B and PVT1 rearrangement occur frequently in highly aggressive B-cell lymphomas harboring 8q24 abnormality. Leuk Lymphoma. 2013; 54: 2760-4.

15. Northcott PA, Shih DJ, Peacock J, Garzia L, Morrissy AS, Zichner T, et al. Subgroup-specific structural variation across 1,000 medulloblastoma genomes. Nature. 2012; 488: 49-56.

16. Beck-Engeser GB, Lum AM, Huppi K, Caplen NJ, Wang BB, Wabl M. Pvt1-encoded microRNAs in oncogenesis. Retrovirology. 2008; 5: 4.

17. Tseng YY, Moriarity BS, Gong W, Akiyama R, Tiwari A, Kawakami H, et al. PVT1 dependence in cancer with MYC copy-number increase. Nature. 2014; 512: $82-6$. 
18. Carramusa L, Contino F, Ferro A, Minafra L, Perconti G, Giallongo A, et al. The PVT-1 oncogene is a Myc protein target that is overexpressed in transformed cells. J Cell Physiol. 2007; 213: 511-8.

19. Zhou DD, Liu XF, Lu CW. Long non-coding RNA PVT1: Emerging biomarker in digestive system cancer. Cell Prolif. 2017; 50: e12398.

20. Wang F, Yuan JH, Wang SB, Yang F, Yuan SX, Ye C, et al. Oncofetal long noncoding RNA PVT1 promotes proliferation and stem cell-like property of hepatocellular carcinoma cells by stabilizing NOP2. Hepatology. 2014; 60: 1278-90.

21. Liu HT, Fang L, Cheng YX, Sun Q. LncRNA PVT1 regulates prostate cancer cell growth by inducing the methylation of miR-146a. Cancer Med. 2016; 5: $3512-9$.

22. Hindorff LA, Sethupathy P, Junkins HA, Ramos EM, Mehta JP, Collins FS, et al. Potential etiologic and functional implications of genome-wide association loci for human diseases and traits. Proc NatI Acad Sci U S A. 2009; 106: 9362-7.

23. Yin Z, Cui Z, Li H, Li J, Zhou B. Polymorphisms in the H19 gene and the risk of lung Cancer among female never smokers in Shenyang, China. BMC Cancer. 2018; 18: 893.

24. Hu L, Chen SH, Lv QL, Sun B, Qu Q, Qin CZ, et al. Clinical Significance of Long Non-Coding RNA CASC8 rs10505477 Polymorphism in Lung Cancer Susceptibility, Platinum-Based Chemotherapy Response, and Toxicity. Int J Environ Res Public Health. 2016; 13: 545

25. Enciso-Mora V, Broderick P, Ma Y, Jarrett RF, Hjalgrim H, Hemminki K, et al. A genome-wide association study of Hodgkin's lymphoma identifies new susceptibility loci at 2p16.1 (REL), 8q24.21 and 10p14 (GATA3). Nat Genet. 2010; 42: 1126-30.

26. Wolpin BM, Rizzato C, Kraft P, Kooperberg C, Petersen GM, Wang Z, et al. Genome-wide association study identifies multiple susceptibility loci for pancreatic cancer. Nat Genet. 2014; 46: 994-1000.

27. Skibola CF, Berndt SI, Vijai J, Conde L, Wang Z, Yeager M, et al. Genome-wide association study identifies five susceptibility loci for follicular lymphoma outside the HLA region. Am J Hum Genet. 2014; 95: 462-71.

28. Ren Y, Huang W, Weng G, Cui P, Liang H, Li Y. LncRNA PVT1 promotes proliferation, invasion and epithelial-mesenchymal transition of renal cell carcinoma cells through downregulation of miR-16-5p. Onco Targets Ther. 2019; 12: 2563-75

29. Yang T, Zhou H, Liu P, Yan L, Yao W, Chen K, et al. IncRNA PVT1 and its splicing variant function as competing endogenous RNA to regulate clear cell renal cell carcinoma progression. Oncotarget. 2017; 8: 85353-67.

30. Li W, Zheng Z, Chen H, Cai Y, Xie W. Knockdown of long non-coding RNA PVT1 induces apoptosis and cell cycle arrest in clear cell renal cell carcinoma through the epidermal growth factor receptor pathway. Oncol Lett. 2018; 15: 7855-63

31. Yang J, Li C, Mudd A, Gu X. LncRNA PVT1 predicts prognosis and regulates tumor growth in prostate cancer. Biosci Biotechnol Biochem. 2017; 81: 2301-6.

32. Wan B, Wu HY, Lv DJ, Zhou XM, Zhong LR, Lei B, et al. Downregulation of lncRNA PVT1 expression inhibits proliferation and migration by regulating p38 expression in prostate cancer. Oncol Lett. 2018; 16: 5160-6.

33. Chang Z, Cui J, Song Y. Long noncoding RNA PVT1 promotes EMT via mediating microRNA-186 targeting of Twist1 in prostate cancer. Gene. 2018; 654: $36-42$.

34. Xu MD, Wang Y, Weng W, Wei P, Qi P, Zhang Q, et al. A Positive Feedback Loop of IncRNA-PVT1 and FOXM1 Facilitates Gastric Cancer Growth and Invasion. Clin Cancer Res. 2017; 23: 2071-80.

35. Kong R, Zhang EB, Yin DD, You LH, Xu TP, Chen WM, et al. Long noncoding RNA PVT1 indicates a poor prognosis of gastric cancer and promotes cell proliferation through epigenetically regulating p15 and p16. Mol Cancer. 2015; 14: 82 .

36. Yuan CL, Li H, Zhu L, Liu Z, Zhou J, Shu Y. Aberrant expression of long noncoding RNA PVT1 and its diagnostic and prognostic significance in patients with gastric cancer. Neoplasma. 2016; 63: 442-9.

37. Huang T, Liu HW, Chen JQ, Wang SH, Hao LQ, Liu M, et al. The long noncoding RNA PVT1 functions as a competing endogenous RNA by sponging miR-186 in gastric cancer. Biomed Pharmacother. 2017; 88: 302-8.

38. Zhao J, Du P, Cui P, Qin Y, Hu C, Wu J, et al. LncRNA PVT1 promotes angiogenesis via activating the STAT3/VEGFA axis in gastric cancer. Oncogene. 2018; 37: 4094-109.

39. Li T, Meng XL, Yang WQ. Long Noncoding RNA PVT1 Acts as a "Sponge" to Inhibit microRNA-152 in Gastric Cancer Cells. Dig Dis Sci. 2017; 62: 3021-8.

40. Zhang Y, Yang G, Luo Y. Long non-coding RNA PVT1 promotes glioma cell proliferation and invasion by targeting miR-200a. Exp Ther Med. 2019; 17: $1337-45$

41. Zou H, Wu LX, Yang Y, Li S, Mei Y, Liu YB, et al. IncRNAs PVT1 and HAR1A are prognosis biomarkers and indicate therapy outcome for diffuse glioma patients. Oncotarget. 2017; 8: 78767-80.

42. Fu C, Li D, Zhang $X$, Liu N, Chi G, Jin X. LncRNA PVT1 Facilitates Tumorigenesis and Progression of Glioma via Regulation of MiR-128-3p/GREM1 Axis and BMP Signaling Pathway. Neurotherapeutics. 2018; 15: 1139-57.

43. Yang A, Wang H, Yang X. Long non-coding RNA PVT1 indicates a poor prognosis of glioma and promotes cell proliferation and invasion via target EZH2. Biosci Rep. 2017; 37: BSR20170871.

44. Ma Y, Wang P, Xue Y, Qu C, Zheng J, Liu X, et al. PVT1 affects growth of glioma microvascular endothelial cells by negatively regulating miR-186. Tumour Biol. 2017; 39: 1010428317694326.
45. Zhang R, Li J, Yan X, Jin K, Li W, Liu X, et al. Long Noncoding RNA Plasmacytoma Variant Translocation 1 (PVT1) Promotes Colon Cancer Progression via Endogenous Sponging miR-26b. Med Sci Monit. 2018; 24: 8685-92.

46. Chai J, Guo D, Ma W, Han D, Dong W, Guo H, et al. A feedback loop consisting of RUNX2/LncRNA-PVT1/miR-455 is involved in the progression of colorectal cancer. Am J Cancer Res. 2018; 8: 538-50.

47. Yu X, Zhao J, He Y. Long non-coding RNA PVT1 functions as an oncogene in human colon cancer through miR-30d-5p/RUNX2 axis. J BUON. 2018; 23 : 48-54

48. Fan $\mathrm{H}$, Zhu JH, Yao XQ. Long non-coding RNA PVT1 as a novel potential biomarker for predicting the prognosis of colorectal cancer. Int J Biol Markers. 2018; 515-22.

49. Li X, Chen W, Wang H, Wei Q, Ding X, Li W. Amplification and the clinical significance of circulating cell-free DNA of PVT1 in breast cancer. Oncol Rep. 2017; 38: 465-71.

50. Wang Y, Zhou J, Wang Z, Wang P, Li S. Upregulation of SOX2 activated LncRNA PVT1 expression promotes breast cancer cell growth and invasion. Biochem Biophys Res Commun. 2017; 493: 429-36.

51. Tang J, Li Y, Sang Y, Yu B, Lv D, Zhang W, et al. LncRNA PVT1 regulates triple-negative breast cancer through KLF5/beta-catenin signaling. Oncogene. 2018; 37: 4723-34.

52. Sarver AL, Murray CD, Temiz NA, Tseng YY, Bagchi A. MYC and PVT1 synergize to regulate RSPO1 levels in breast cancer. Cell Cycle. 2016; 15: 881-5.

53. Chen $\mathrm{Y}, \mathrm{Du} \mathrm{H}$, Bao L, Liu W. LncRNA PVT1 promotes ovarian cancer progression by silencing miR-214. Cancer Biol Med. 2018; 15: 238-50.

54. Yang Q, Yu Y, Sun Z, Pan Y. Long non-coding RNA PVT1 promotes cell proliferation and invasion through regulating miR-133a in ovarian cancer. Biomed Pharmacother. 2018; 106: 61-7.

55. Liu E, Liu Z, Zhou Y, Mi R, Wang D. Overexpression of long non-coding RNA PVT1 in ovarian cancer cells promotes cisplatin resistance by regulating apoptotic pathways. Int J Clin Exp Med. 2015; 8: 20565-72.

56. Zou MF, Ling J, Wu QY, Zhang CX. Long non-coding RNA PVT1 functions as an oncogene in ovarian cancer via upregulating SOX2. Eur Rev Med Pharmacol Sci. 2018; 22: 7183-8.

57. Tian Z, Cao S, Li C, Xu M, Wei H, Yang H, et al. LncRNA PVT1 regulates growth, migration, and invasion of bladder cancer by miR-31/ CDK1. J Cell Physiol. 2019; 234: 4799-811.

58. Song J, Wu X, Liu F, Li M, Sun Y, Wang Y, et al. Long non-coding RNA PVT1 promotes glycolysis and tumor progression by regulating miR-497/HK2 axis in osteosarcoma. Biochem Biophys Res Commun. 2017; 490: 217-24.

59. Zhou Q, Chen F, Zhao J, Li B, Liang Y, Pan W, et al. Long non-coding RNA PVT1 promotes osteosarcoma development by acting as a molecular sponge to regulate miR-195. Oncotarget. 2016; 7: 82620-33.

60. Zhao L, Kong H, Sun H, Chen Z, Chen B, Zhou M. LncRNA-PVT1 promotes pancreatic cancer cells proliferation and migration through acting as a molecular sponge to regulate miR-448. J Cell Physiol. 2018; 233: 4044-55.

61. Wu BQ, Jiang $\mathrm{Y}$, Zhu F, Sun DL, He XZ Long Noncoding RNA PVT1 Promotes EMT and Cell Proliferation and Migration Through Downregulating p21 in Pancreatic Cancer Cells. Technol Cancer Res Treat. 2017; 16: 819-27.

62. Iden M, Fye S, Li K, Chowdhury T, Ramchandran R, Rader JS. The IncRNA PVT1 Contributes to the Cervical Cancer Phenotype and Associates with Poor Patient Prognosis. PloS one. 2016; 11: e0156274.

63. Zhang S, Zhang G, Liu J. Long noncoding RNA PVT1 promotes cervical cancer progression through epigenetically silencing miR-200b. APMIS. 2016; 124: 649-58.

64. He Y, Jing Y, Wei F, Tang Y, Yang L, Luo J, et al. Long non-coding RNA PVT1 predicts poor prognosis and induces radioresistance by regulating DNA repair and cell apoptosis in nasopharyngeal carcinoma. Cell Death Dis. 2018; 9: 235

65. Li PD, Hu JL, Ma C, Ma H, Yao J, Chen LL, et al. Upregulation of the long non-coding RNA PVT1 promotes esophageal squamous cell carcinoma progression by acting as a molecular sponge of miR-203 and LASP1. Oncotarget. 2017; 8: 34164-76.

66. Lan T, Yan X, Li Z, Xu X, Mao Q, Ma W, et al. Long non-coding RNA PVT1 serves as a competing endogenous RNA for miR-186-5p to promote the tumorigenesis and metastasis of hepatocellular carcinoma. Tumour Biol. 2017; 39: 1010428317705338

67. Gou X, Zhao X, Wang Z. Long noncoding RNA PVT1 promotes hepatocellular carcinoma progression through regulating miR-214. Cancer Biomark. 2017; 20: $511-9$.

68. Guo J, Hao C, Wang C, Li L . Long noncoding RNA PVT1 modulates hepatocellular carcinoma cell proliferation and apoptosis by recruiting EZH2. Cancer Cell Int. 2018; 18: 98.

69. Huang T, Wang G, Yang L, Peng B, Wen Y, Ding G, et al. Transcription Factor YY1 Modulates Lung Cancer Progression by Activating lncRNA-PVT1. DNA Cell Biol. 2017; 36: 947-58

70. Li H, Chen S, Liu J, Guo X, Xiang X, Dong T, et al. Long non-coding RNA PVT1-5 promotes cell proliferation by regulating miR-126/SLC7A5 axis in lung cancer. Biochem Biophys Res Commun. 2018; 495: 2350-5.

71. Shi T, Gao G, Cao Y. Long Noncoding RNAs as Novel Biomarkers Have a Promising Future in Cancer Diagnostics. Dis Markers. 2016; 2016: 9085195.

72. Qiu MT, Hu JW, Yin R, Xu L. Long noncoding RNA: an emerging paradigm of cancer research. Tumour Biol. 2013; 34: 613-20. 
73. Zhang H, Chen Z, Wang X, Huang Z, He Z, Chen Y. Long non-coding RNA: a new player in cancer. J Hematol Oncol. 2013; 6: 37.

74. Jin Y, Feng SJ, Qiu S, Shao N, Zheng JH. LncRNA MALAT1 promotes proliferation and metastasis in epithelial ovarian cancer via the PI3K-AKT pathway. Eur Rev Med Pharmacol Sci. 2017; 21: 3176-84.

75. Luo J, Chen J, Li H, Yang Y, Yun H, Yang S, et al. LncRNA UCA1 promotes the invasion and EMT of bladder cancer cells by regulating the miR-143/HMGB1 pathway. Oncol Lett. 2017; 14: 5556-62.

76. Chen W, Zhu H, Yin L, Wang T, Wu J, Xu J, et al. IncRNA-PVT1 Facilitates Invasion Through Upregulation of MMP9 in Nonsmall Cell Lung Cancer Cell. DNA Cell Biol. 2017; 36: 787-93.

77. Wan L, Sun M, Liu GJ, Wei CC, Zhang EB, Kong R, et al. Long Noncoding RNA PVT1 Promotes Non-Small Cell Lung Cancer Cell Proliferation through Epigenetically Regulating LATS2 Expression. Mol Cancer Ther. 2016; 15: 1082-94.

78. Zhang Z, Zhu Z, Zhang B, Li W, Li X, Wu X, et al. Frequent mutation of rs13281615 and its association with PVT1 expression and cell proliferation in breast cancer. J Genet Genomics. 2014; 41: 187-95.

79. Zhang W, Xiao J, Lu X, Liu T, Jin X, Xiao Y. PVT1 (rs13281615) and miR-146a (rs2910164) polymorphisms affect the prognosis of colon cancer by regulating COX2 expression and cell apoptosis. J Cell Physiol. 2019; 234: 17538-48.

80. Moschovis D, Vasilaki E, Tzouvala M, Karamanolis G, Katifelis H, Legaki E, et al. Association between genetic polymorphisms in long non-coding RNAs and pancreatic cancer risk. Cancer Biomark. 2019; 24: 117-23. 